\title{
Regulation of Self-renewal and Differentiation in the Drosophila Nervous System
}

\author{
T.D. Southall, B. EgGer, K.S. Gold, AND A.H. BRAND \\ The Gurdon Institute and Department of Physiology, Development, and Neuroscience, \\ University of Cambridge, Cambridge CB2 1QN, United Kingdom
}

\begin{abstract}
Stem cells can divide symmetrically to generate two similar daughter cells and expand the stem cell pool or asymmetrically to self-renew and generate differentiating daughter cells. The proper balance between symmetric and asymmetric division is critical for the generation and subsequent repair of tissues. Furthermore, unregulated stem cell division has been shown to result in tumorous overgrowth. The Drosophila nervous system has proved to be a fruitful model system for studying the biology of neural stem cell division and uncovering the molecular mechanisms that, when disrupted, can lead to tumor formation. We are using the Drosophila embryonic and larval nervous systems as models to study the regulation of symmetric and asymmetric stem cell division.
\end{abstract}

Stem cells have the capacity to renew themselves at each division while producing a continuous supply of differentiating daughters for the generation, and subsequent repair, of tissues (for review, see Weissman et al. 2001). These two aspects of stem cell biology must be carefully balanced during development to ensure that organs of the right size and tissue composition are formed. The spatially and temporally regulated differentiation of multipotent stem cells generates the enormous cellular diversity seen in the nervous system. A common strategy that stem cells use to generate cellular diversity is asymmetric cell division, where the unequal distribution of cell-fate determinants leads to the production of daughter cells with different fates (for review, see Morrison and Kimble 2006; Doe 2008; Gonczy 2008; Knoblich 2008). The Drosophila nervous system has proved to be a fertile ground not only for studying the asymmetric divisions of stem cells (Wu et al. 2008), but also, more recently, for understanding how unregulated division in a stem cell lineage can lead to tumorigenesis (Caussinus and Gonzalez 2005; Bello et al. 2006; Betschinger et al. 2006; Choksi et al. 2006; Lee et al. 2006a). Here, we focus on two key aspects of neural stem cell regulation: how the transition from proliferation to differentiation is achieved within a pool of stem cells and how the switch from self-renewal to differentiation is balanced within a single stem cell division.

\section{SYMMETRIC VERSUS ASYMMETRIC DIVISION IN THE CENTRAL NERVOUS SYSTEM}

Stem cells can proliferate through symmetric division, which leads to similar daughter cells, or they can divide asymmetrically to self-renew the mother cell and generate a differentiating daughter cell. The correct ratio between symmetric and asymmetric stem cell divisions is crucial for achieving the proper size of an organ and for the timely differentiation of a tissue. Failure to maintain this balance, or the incorrect activation of either division mode, can lead to tumorous overgrowth or to premature differentiation (Morrison and Kimble 2006).

The importance of tight developmental controls over asymmetric division has been demonstrated in Drosophila by a number of studies showing that when the machinery that regulates asymmetric divisions is disrupted, neuroblasts proliferate aberrantly and form tumors (Albertson and Doe 2003; Caussinus and Gonzalez 2005; Bello et al. 2006; Betschinger et al. 2006; Choksi et al. 2006; Lee et al. 2006a,b). Less is known about the regulation of symmetric, proliferative stem cell division, which serves to increase a stem cell pool during normal development, nor how the transition from symmetric to asymmetric division is controlled. Such a transition can be seen in the developing mammalian cortex, where neuroepithelial cells initially proliferate symmetrically and then convert to radial glial cells (the main progenitor type) during neurogenesis (Gotz and Huttner 2005). The Drosophila larval optic lobe provides an elegant and tractable system for studying how the switch from one mode of division to another occurs.

The optic lobe generates the visual processing centers of the adult brain. It arises from two proliferative neuroepithelia in the larva, known as the inner and outer proliferation centers (IPC and OPC, respectively). During larval development, two morphologically and molecularly distinct cell populations can be identified in the OPC: lateral, symmetrically dividing neuroepithelial cells and medial, asymmetrically dividing medulla neuroblasts (Fig. 1) (White and Kankel 1978; Hofbauer and CamposOrtega 1990; Ceron et al. 2001; Egger et al. 2007). Clonal analysis has shown that the symmetrically dividing neuroepithelial progenitors transform into asymmetrically dividing medulla neuroblasts at the medial edge of the OPC (Fig. 2) (Egger et al. 2007).

The transition from neuroepithelial cell to neuroblast occurs in a temporally regulated progression (Egger et al. 2007). The molecular mechanisms underlying this transition remain largely unknown, with the exception of one 


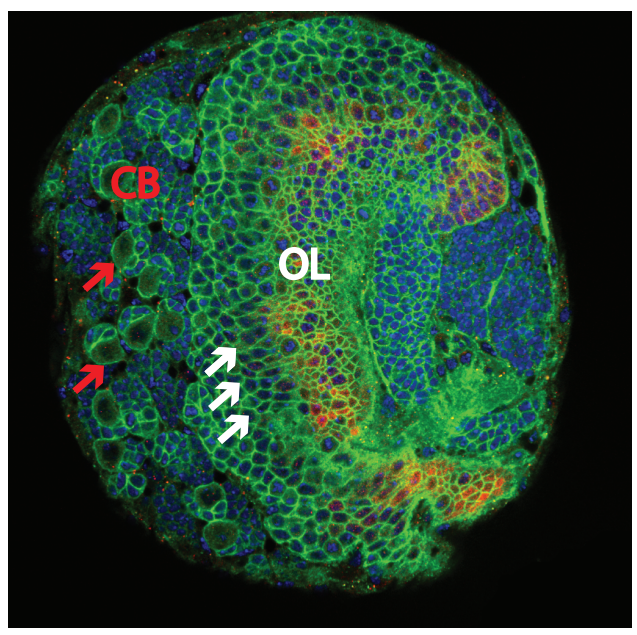

Figure 1. Neural stem cells in the larval brain. The symmetrically dividing neuroepithelial cells and asymmetrically dividing neuroblasts of the optic lobe (OL, white arrows) can be seen on the right-hand side of the brain lobe, whereas, on the left, the large neuroblasts of the central brain $(\mathrm{CB}$, red arrows) are evident. (Green) Dlg; (red) l'sc-mRFP1; (blue) DNA.

recent study (Yasugi et al. 2008). Yasugi and colleagues have shown that the transition from symmetrically dividing neuroepithelial cells to asymmetrically dividing neuroblasts coincides with expression of the proneural gene lethal of scute ( $l$ 'sc) in the most medial epithelial cells. The transformation of neuroepithelial cells to neuroblasts is driven by a "proneural wave" of L'sc expression that sweeps across the neuroepithelium from medial to lateral. Progession of this proneural wave is negatively regulated by the Janus kinase-signal transducer and activator of transcription (JAK/STAT) pathway, whose ligand, Unpaired (Upd), signals from the most lateral neuroepithelial cells (Yasugi et al. 2008).

To identify potential regulators of the symmetric-toasymmetric transition, we characterized the transcriptional profiles of neuroepithelial cells and medulla neuroblasts. We devised a method by which to label and extract neuroepithelial cells and neuroblasts from the brains of live late third-instar larvae. Neuroepithelial and neuroblast cDNA samples were collected and directly compared on Drosophila whole transcriptome oligonucleotide microarrays. Statistical analysis of the data yielded nearly 200 genes that are significantly differentially expressed between neuroepithelial cells and neuroblasts. Several distinct classes of genes are strikingly enriched in the optic lobe neuroepithelium: the Delta/Notch and Hedgehog signaling pathways, proneural genes, and a number of genes with a role in retinal determination.

\section{SELF-RENEWAL VERSUS DIFFERENTIATION IN THE CENTRAL NERVOUS SYSTEM}

Drosophila neuroblasts divide in a regenerative fashion, producing a large daughter cell that self-renews and a smaller daughter cell (a ganglion mother cell [GMC]) that divides only once to give two postmitotic neurons or glial cells. This asymmetry in cell fate is achieved by the unequal partitioning of determinants (for review, see Morrison and Kimble 2006; Doe 2008; Gonczy 2008; Knoblich 2008; Zhong and Chia 2008). At each neuroblast division, cellfate determinants, such as the phosphotyrosine-binding (PTB) domain protein Numb, the homeodomain transcription factor Prospero, and the NHL-domain protein Brain Tumour (Brat), are localized to the basal cortex by the adapter proteins Partner of Numb (Pon) and Miranda. When neuroblasts divide, only the basal daughter cell (the GMC) inherits Miranda, Pon, and their cargo.

We have been studying the role of Prospero in asymmetric division in regulating self-renewal and differentiation. Prospero is conserved in vertebrates, where the Prox (for Prospero-related homeobox) family of atypical homeodomain transcription factors appears to have a role in initiating the differentiation of progenitors in various

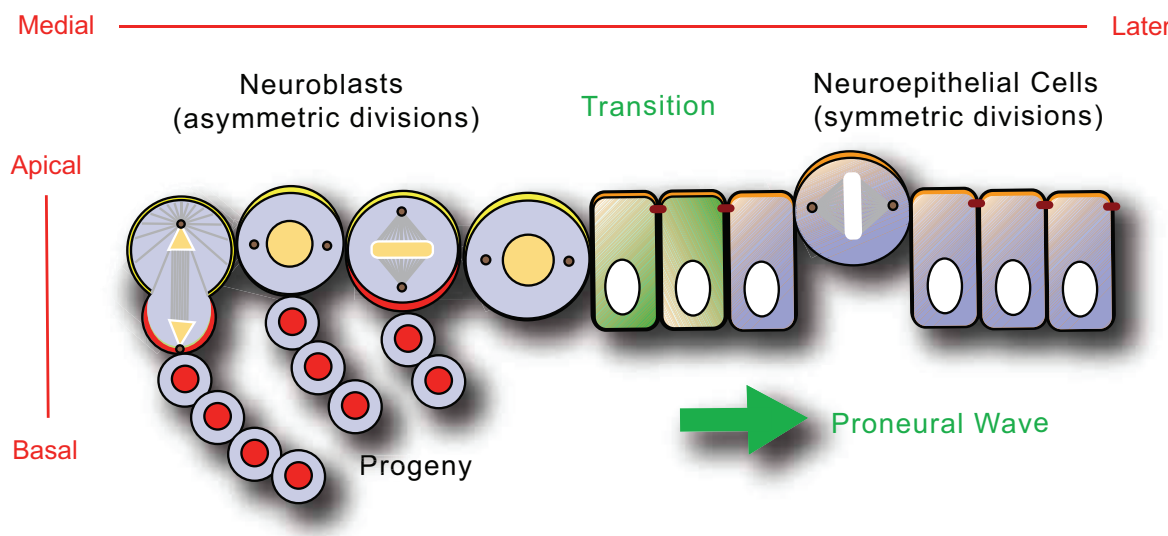

Figure 2. Neuroepithelial-to-neuroblast transition in the larval optic lobe. At the medial edge of the optic lobe, columnar neuroepithelial cells undergo a transition to neuroblasts. Neuroepithelial cells divide symmetrically, with horizontal spindle orientation, which results in the expansion of the progenitor pool. Medial neuroblasts divide asymmetrically, with vertical spindle orientation, and bud off smaller ganglion mother cells (GMCs) toward the medulla cortex. Most medial neuroepithelial cells (green) transiently express the proneural gene, lethal of scute ( $l$ 'sc). This expression sweeps across the epithelium in the form of a proneural wave, from medial to lateral, as more neuroblasts are generated. (Adapted from Egger et al. 2007; Yasugi et al. 2008.) 
tissues (Torii et al. 1999; Wigle et al. 2002; Dyer et al. 2003; Pistocchi et al. 2008). Prox1 is also associated with several reported cases of cancer progression (Versmold et al. 2007; Yoshimoto et al. 2007; Dudas et al. 2008; Petrova et al. 2008).

Prospero is transcribed in the neuroblast but not the GMC (Broadus et al. 1998). Prospero protein and its mRNA are anchored at the cortex of the neuroblast by the adapter protein Miranda (Ikeshima-Kataoka et al. 1997; Shen et al. 1997, 1998; Matsuzaki et al. 1998; Schuldt et al. 1998). Once segregated to the GMC by Miranda, Prospero is released from the cortex and enters the GMC nucleus. The nuclear localization of Prospero is one of the first molecular differences between a self-renewing neuroblast and a GMC (Hirata et al. 1995; Knoblich et al. 1995; Spana and Doe 1995). Therefore, identifying the genes regulated by Prospero should provide important insights into the genetic networks governing the switch from a stem cell to a differentiating cell.

To determine the sites to which Prospero binds throughout the genome, we used the in vivo chromatin profiling technique DamID (van Steensel and Henikoff 2000; van Steensel et al. 2001). In brief, DamID involves fusing any DNA or chromatin-associated protein of interest to a bacterial DNA adenine methyltransferase (Dam). When the fusion protein binds to its native site in the genome, the methylase leaves a unique methylation tag on the sequence GATC. Genomic DNA is then extracted and digested with DpnI, which cuts specifically at methylated GATC sites. The tagged sequences can then be isolated and identified on DNA tiling microarrays.

We generated transgenic embryos expressing a DamProspero fusion protein, or Dam alone to control for nonspecific methylation, and collected genomic DNA material from stage-10-11 embryos. Following digestion with DpnI and ligation-mediated polymerase chain reaction (PCR) amplification of the methylated regions of DNA, the DNA was hybridized to full-genome tiling microarrays covering the whole euchromatic genome (Fig. 3A) (Choksi et al. 2006). The data from four biological replicates identified 1602 Prospero-binding sites. Of these, 1066 contain a previously published Prospero-consensus-binding site (Cook
A
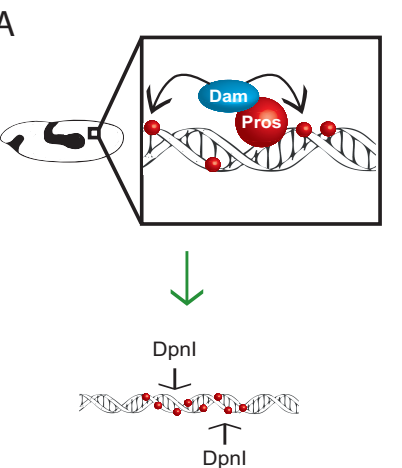

$\downarrow$

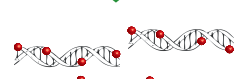

2 T. T0

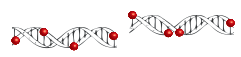

$\downarrow$

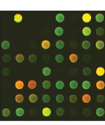

$\downarrow$

Putative Target

Genes
B

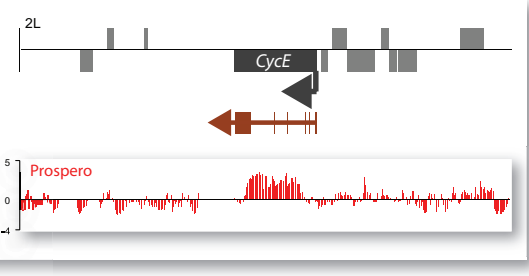

D

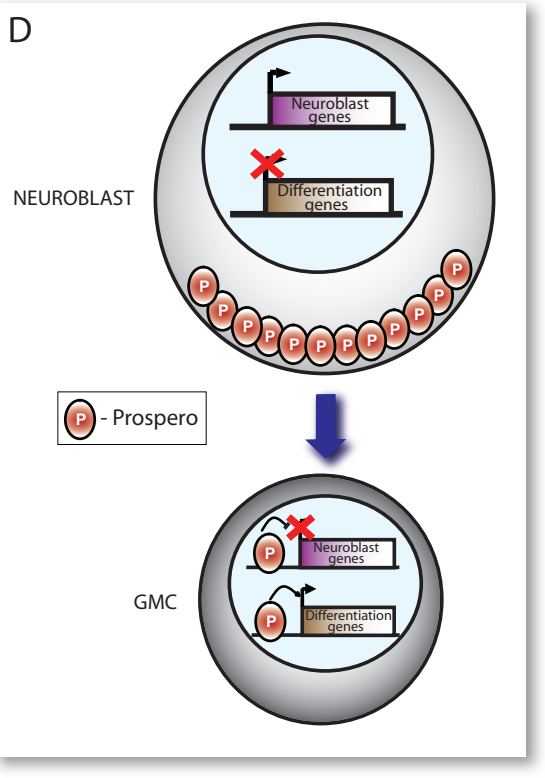

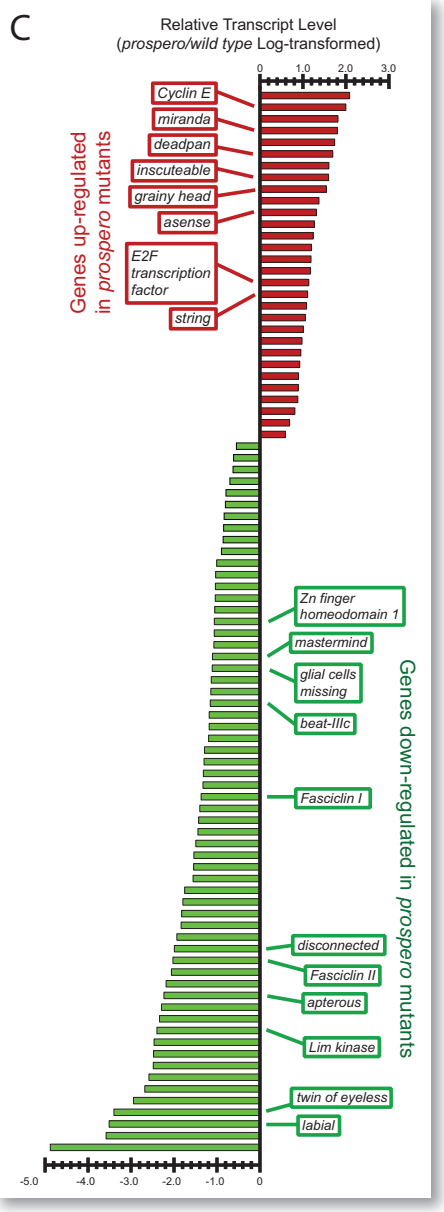

Figure 3. Genome-wide mapping of Prospero-binding sites. (A) Schematic diagram of the DamID technique. (B) Prospero binding at the $C y c E$ locus. (Gray and black boxes) Genes; (brown boxes) CycE exons. Bar heights are proportional to the average of normalized log-transformed ratio of intensities from four replicate DamID in vivo binding-site-mapping experiments. $(C)$ High-resolution gene expression profiling of cells from the ventral nerve cord of prospero mutants. (Red) Genes that are up-regulated; (green) genes that are down-regulated. $(D)$ Model showing Prospero's segregation from the neuroblast to the GMC, where it enters the nucleus to repress neuroblast genes and activate differentiation genes. 
et al. 2003), corresponding to 730 genes (Choksi et al. 2006). An example of Prospero binding to the intronic regions of Cyclin E $(C y c E)$ is shown in Figure 3B.

An unbiased gene ontology analysis of these genes using GOtoolbox (Martin et al. 2004) highlighted cellfate commitment, nervous system development, and regulation of transcription as the most enriched classes of biological function. This correlates with the known function and expression pattern of Prospero. Furthermore, Prospero binds to $41 \%$ of all annotated neuroblast genes and key regulators of the cell cycle, including Cyclin $E$ (Fig. 3B), the $c d c 25$ homolog string, and E2F.

Expression of Prospero is maintained in glial cells but not in neurons, where the protein is absent (Spana and Doe 1995). Consistent with this, Prospero directly binds $45 \%$ of genes annotated to have a role in gliogenesis. However, to our surprise, we find that Prospero also binds to many genes involved in the terminal differentiation of neurons. These include $19 \%$ of all annotated neuronal differentiation genes, including the adhesion molecule FasciclinII (Lin et al. 1994) and the axon guidance molecule Netrin-B (Mitchell et al. 1996).

DamID provides information on whether a gene is bound by a transcription factor but not whether it is activated or repressed. To elucidate how Prospero regulates its target genes, we performed transcriptional profiling of neuroblasts and GMCs from wild-type and prospero mutant embryos. Approximately 100 cells were isolated from the ventral nerve cords of embryos using a glass capillary. The cells were expelled into lysis buffer, and cDNA was generated by reverse transcription and PCR amplification (Iscove et al. 2002; Choksi et al. 2006). These cDNA libraries were then compared on fullgenome oligonucleotide microarrays.

We identified 91 genes that are directly bound by Prospero and show a significant change in transcription (Fig. 3C) (Choksi et al. 2006). Many genes involved in neuroblast cell-fate determination (asense, deadpan, miranda, inscuteable) and in cell cycle regulation (Cyclin $E$ and string) show increased levels in a prospero mutant background, consistent with their being repressed by Prospero. Conversely, genes with a role in neuronal differentiation show decreased transcription in prospero mutants, indicating that Prospero is required for their transcription.

\section{GMCS ARE TRANSFORMED INTO NEURAL STEM CELLS IN PROSPERO MUTANTS}

If Prospero is key in promoting differentiation, then, in the absence of Prospero, neuroblasts should give rise to two self-renewing neuroblast-like cells. We followed the division pattern of individual neuroblasts in prospero mutant embryos by labeling with the lipophilic dye DiI (Choksi et al. 2006). Wild-type neuroblasts generate, on average, 16.2 cells that exhibit extensive axonal outgrowth (Fig. 4, left). However, prospero mutant neuroblasts produce an average of 31.8 cells with few, if any, projections (Fig. 4, right). Thus, prospero mutant neuroblasts produce much larger clones of cells with no axonal projections,

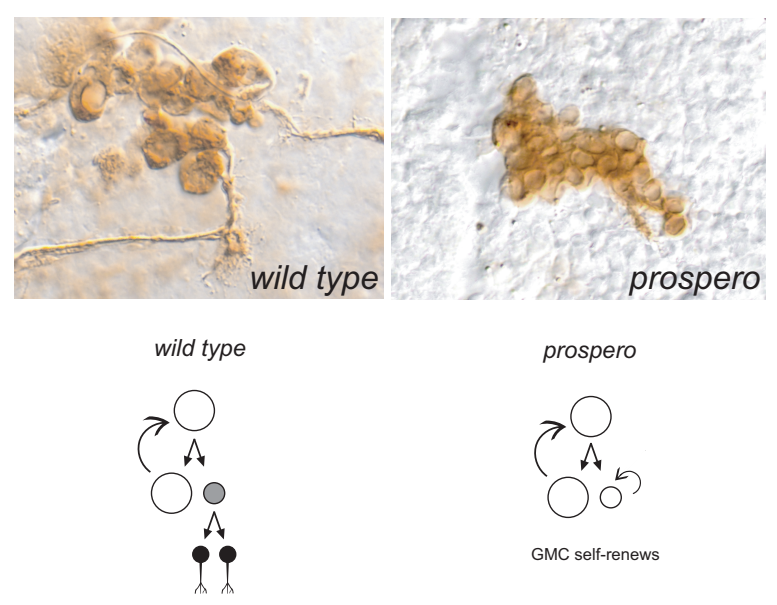

Figure 4. Neuroblasts overproliferate and fail to differentiate in prospero mutants. (Left) Wild-type clones, from a single S1 or S2 neuroblast labeled with DiI, extend axonal projections that often exit the central nervous system. (Right) prospero mutant clones give, on average, twice as many cells without axonal projections. (Left schematic diagram) Wild-type neuroblasts (white) divide in a self-renewing manner to produce a neuroblast and a GMC (gray). The GMC divides only once to produce two neurons or glial cells (black). (Right schematic diagram) In a prospero mutant, GMCs divide in a self-renewing manner.

suggesting that neural cells in prospero mutants undergo extra divisions and fail to differentiate. We followed the division pattern of individual GMCs in prospero mutant embryos by first labeling a single neuroblast with DiI. After the first cell division, we removed the neuroblast, leaving only a single labeled first-born GMC. Whereas wild-type GMCs give rise to just two daughter cells that extend axons, prospero mutant GMCs give up to seven cells that continue to express neuroblast markers and exhibit few, if any, signs of differentiation. We conclude that prospero mutant neuroblasts divide to give two stemcell-like daughters. GMCs, which would normally terminate cell division and differentiate, are transformed into self-renewing neural stem cells that generate undifferentiated clones, or tumors. These results are similar to what has been observed in the larval brain, where clones of cells lacking Prospero or Brat undergo extensive cell division to generate undifferentiated tumors (Bello et al. 2006; Betschinger et al. 2006; Lee et al. 2006a).

Our data demonstrate that Prospero represses neural stem cell genes and is required for the activation of neuronal differentiation genes, suggesting that it acts as a binary switch between self-renewal and differentiation in neural stem cells. Consequently, when Prospero is absent, the daughter cell (to which Prospero is normally segregated) is no longer programmed to differentiate but continues to behave like a stem cell, undergoing further divisions and producing tumors.

Prospero is also present in the daughter cells of Drosophila midgut stem cells (Micchelli and Perrimon 2006; Ohlstein and Spradling 2006), suggesting that it may have a more universal role in regulating stem cell differentiation. 


\section{REFERENCES}

Albertson, R. and Doe, C.Q. 2003. Dlg, Scrib and Lgl regulate neuroblast cell size and mitotic spindle asymmetry. Nat. Cell Biol. 5: 166-170.

Bello, B., Reichert, H., and Hirth, F. 2006. The brain tumor gene negatively regulates neural progenitor cell proliferation in the larval central brain of Drosophila. Development 133: 2639 2648.

Betschinger, J., Mechtler, K., and Knoblich, J.A. 2006. Asymmetric segregation of the tumor suppressor brat regulates selfrenewal in Drosophila neural stem cells. Cell 124: 1241-1253.

Broadus, J., Fuerstenberg, S., and Doe, C.Q. 1998. Staufen-dependent localization of prospero mRNA contributes to neuroblast daughter-cell fate. Nature 391: 792-795.

Caussinus, E. and Gonzalez, C. 2005. Induction of tumor growth by altered stem-cell asymmetric division in Drosophila melanogaster. Nat. Genet. 37: 1125-1129.

Ceron, J., Gonzalez, C., and Tejedor, F.J. 2001. Patterns of cell division and expression of asymmetric cell fate determinants in postembryonic neuroblast lineages of Drosophila. Dev. Biol. 230: $125-138$.

Choksi, S.P., Southall, T.D., Bossing, T., Edoff, K., de Wit, E., Fischer, B.E., van Steensel, B., Micklem, G., and Brand, A.H. 2006. Prospero acts as a binary switch between self-renewal and differentiation in Drosophila neural stem cells. Dev. Cell 11: 775-789.

Cook, T., Pichaud, F., Sonneville, R., Papatsenko, D., and Desplan, C. 2003. Distinction between color photoreceptor cell fates is controlled by Prospero in Drosophila. Dev. Cell 4: 853864.

Doe, C.Q. 2008. Neural stem cells: Balancing self-renewal with differentiation. Development 135: 1575-1587.

Dudas, J., Mansuroglu, T., Moriconi, F., Haller, F., Wilting, J., Lorf, T., Füzesi, L., and Ramadori, G. 2008. Altered regulation of Prox 1-gene-expression in liver tumors. BMC Cancer 8: 92.

Dyer, M.A., Livesey, F.J., Cepko, C.L., and Oliver, G. 2003. Prox1 function controls progenitor cell proliferation and horizontal cell genesis in the mammalian retina. Nat. Genet. 34: 53 58.

Egger, B., Boone, J.Q., Stevens, N.R., Brand, A.H., and Doe, C.Q. 2007. Regulation of spindle orientation and neural stem cell fate in the Drosophila optic lobe. Neural Dev. 2: 1.

Gonczy, P. 2008. Mechanisms of asymmetric cell division: Flies and worms pave the way. Nat. Rev. Mol. Cell Biol. 9: 355366.

Gotz, M. and Huttner, W.B. 2005. The cell biology of neurogenesis. Nat. Rev. Mol. Cell Biol. 6: 777-788.

Hirata, J., Nakagoshi, H., Nabeshima, Y., and Matsuzaki, F. 1995. Asymmetric segregation of the homeodomain protein Prospero during Drosophila development. Nature 377: 627-630.

Hofbauer, A. and Campos-Ortega, J.A. 1990. Proliferation pattern and early differentiation of the optic lobes in Drosophila melanogaster. Roux's Arch. Dev. Biol. 198: 264-274.

Ikeshima-Kataoka, H., Skeath, J.B., Nabeshima, Y., Doe, C.Q., and Matsuzaki, F. 1997. Miranda directs Prospero to a daughter cell during Drosophila asymmetric divisions. Nature 390: 625-629.

Iscove, N.N., Barbara, M., Gu, M., Gibson, M., Modi, C., and Winegarden, N. 2002. Representation is faithfully preserved in global cDNA amplified exponentially from sub-picogram quantities of mRNA. Nat. Biotechnol. 20: 940-943.

Knoblich, J.A. 2008. Mechanisms of asymmetric stem cell division. Cell 132: 583-597.

Knoblich, J.A., Jan, L.Y., and Jan, Y.N. 1995. Asymmetric segregation of Numb and Prospero during cell division. Nature 377: 624-627.

Lee, C.Y., Wilkinson, B.D., Siegrist, S.E., Wharton, R.P., and Doe, C.Q. 2006a. Brat is a Miranda cargo protein that promotes neuronal differentiation and inhibits neuroblast self-renewal. Dev. Cell 10: 441-449.

Lee, C.Y., Andersen, R.O., Cabernard, C., Manning, L., Tran, K.D., Lanskey, M.J., Bashirullah, A., and Doe, C.Q. 2006b. Drosophila Aurora-A kinase inhibits neuroblast self-renewal by regulating aPKC/Numb cortical polarity and spindle orientation. Genes Dev. 20: 3464-3474.

Lin, D.M., Fetter, R.D., Kopczynski, C., Grenningloh, G., and Goodman, C.S. 1994. Genetic analysis of fasciclin II in Drosophila: Defasciculation, refasciculation and altered fasciculation. Neuron 13: 1055-1069.

Martin, D., Brun, C., Remy, E., Mouren, P., Thieffry, D., and Jacq, B. 2004. GOToolBox: Functional analysis of gene datasets based on Gene Ontology. Genome Biol. 5: R101.

Matsuzaki, F., Ohshiro, T., Ikeshima-Kataoka, H., and Izumi, H. 1998. miranda localizes staufen and prospero asymmetrically in mitotic neuroblasts and epithelial cells in early Drosophila embryogenesis. Development 125: 4089-4098.

Micchelli, C.A. and Perrimon, N. 2006. Evidence that stem cells reside in the adult Drosophila midgut epithelium. Nature 439: 475-479.

Mitchell, K.J., Doyle, J.L., Serafini, T., Kennedy, T.E., TessierLavigne, M., Goodman, C.S., and Dickson, B.J. 1996. Genetic analysis of Netrin genes in Drosophila: Netrins guide CNS commissural axons and peripheral motor axons. Neuron 17: 203-215.

Morrison, S.J. and Kimble, J. 2006. Asymmetric and symmetric stem-cell divisions in development and cancer. Nature 441: 1068-1074.

Ohlstein, B. and Spradling, A. 2006. The adult Drosophila posterior midgut is maintained by pluripotent stem cells. Nature 439: 470-474.

Petrova, T.V., Nykänen, A., Norrmén, C., Ivanov, K.I., Andersson, L.C., Haglund, C., Puolakkainen, P., Wempe, F., von Melchner, H., Gradwohl, G., et al. 2008. Transcription factor PROX1 induces colon cancer progression by promoting the transition from benign to highly dysplastic phenotype. Cancer Cell 13: 407-419.

Pistocchi, A., Gaudenzi, G., Carra, S., Bresciani, E., Del Giacco, L., and Cotelli, F. 2008. Crucial role of zebrafish proxl in hypothalamic catecholaminergic neurons development. $B M C$ Dev. Biol. 8: 27.

Schuldt, A.J., Adams, J.H., Davidson, C.M., Micklem, D.R., Haseloff, J., St Johnston, D., and Brand, A.H. 1998. Miranda mediates asymmetric protein and RNA localization in the developing nervous system. Genes Dev. 12: 1847-1857.

Shen, C., Knoblich, J.A., Chan, Y., Jiang, M., Jan, L.Y., and Jan, Y.N. 1998. Miranda as a multidomain adapter linking apically localized Inscuteable and basally localized Staufen and Prospero during asymmetric cell division in Drosophila. Genes Dev. 12: 1837-1846.

Shen, C.P., Jan, L.Y., and Jan, Y.N. 1997. Miranda is required for the asymmetric localization of Prospero during mitosis in Drosophila. Cell 90: 449-458.

Spana, E.P. and Doe, C.Q. 1995. The prospero transcription factor is asymmetrically localized to the cell cortex during neuroblast mitosis in Drosophila. Development 121: 3187-3195.

Torii, M., Matsuzaki, F., Osumi, N., Kaibuchi, K., Nakamura, S., Casarosa, S., Guillemot, F., and Nakafuku, M. 1999. Transcription factors Mash-1 and Prox-1 delineate early steps in differentiation of neural stem cells in the developing central nervous system. Development 126: 443-456.

van Steensel, B. and Henikoff, S. 2000. Identification of in vivo DNA targets of chromatin proteins using tethered Dam methyltransferase. Nat. Biotechnol. 18: 424-428.

van Steensel, B., Delrow, J., and Henikoff, S. 2001. Chromatin profiling using targeted DNA adenine methyltransferase. Nat. Genet. 27: 304-308.

Versmold, B., Felsberg, J., Mikeska, T., Ehrentraut, D., Kohler, J., Hampl, J.A., Rohn, G., Niederacher, D., Betz, B., Hellmich, M., et al. 2007. Epigenetic silencing of the candidate tumor suppressor gene Proxl in sporadic breast cancer. Int. J. Cancer 121: $547-554$.

Weissman, I.L., Anderson, D.J., and Gage, F. 2001. Stem and progenitor cells: Origins, phenotypes, lineage commitments, and transdifferentiations. Annu. Rev. Cell Dev. Biol. 17: 387-403.

White, K. and Kankel, D.R. 1978. Patterns of cell division and cell movement in the formation of the imaginal nervous system in Drosophila melanogaster. Dev. Biol. 65: 296-321. 
Wigle, J.T., Harvey, N., Detmar, M., Lagutina, I., Grosveld, G., Gunn, M.D., Jackson, D.G., and Oliver, G. 2002. An essential role for Proxl in the induction of the lymphatic endothelial cell phenotype. EMBO J. 21: 1505-1513.

Wu, P.S., Egger, B., and Brand, A.H. 2008. Asymmetric stem cell division: Lessons from Drosophila. Semin. Cell Dev. Biol. 19: 283-293.

Yasugi, T., Umetsu, D., Murakami, S., Sato, M., and Tabata, T. 2008. Drosophila optic lobe neuroblasts triggered by a wave of proneural gene expression that is negatively regulated by JAK/STAT. Development 135: 1471-1480.

Yoshimoto, T., Takahashi, M., Nagayama, S., Watanabe, G., Shimada, Y., Sakasi, Y., and Kubo, H. 2007. RNA mutations of proxl detected in human esophageal cancer cells by the shifted termination assay. Biochem. Biophys. Res. Commun. 359: 258-262.

Zhong, W. and Chia, W. 2008. Neurogenesis and asymmetric cell division. Curr. Opin. Neurobiol. 18: 4-11. 


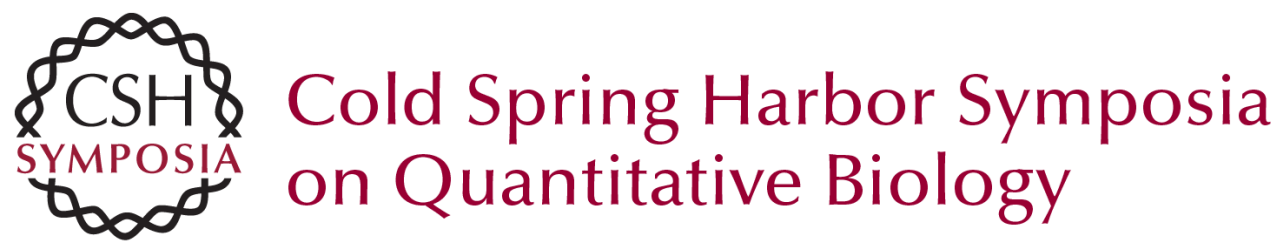

\section{Regulation of Self-renewal and Differentiation in the Drosophila Nervous System}

T.D. Southall, B. Egger, K.S. Gold, et al.

Cold Spring Harb Symp Quant Biol 2008 73: 523-528 originally published online January 15, 2009 Access the most recent version at doi:10.1101/sqb.2008.73.051

References This article cites 46 articles, 10 of which can be accessed free at: http://symposium.cshlp.org/content/73/523.full.html\#ref-list-1

\section{License}

Email Alerting Service top right corner of the article or click here. 